\title{
Quantum Learning: Learn Without Learning
}

Victor Selman, (E-mail: vselman@american.edu), American University

Ruth Corey Selman, Ams Ngo/Dpi Rep To United Nations

Jerry Selman, Quantum Era, Inc.

"I can safely say that nobody understands Quantum Mechanics--"

Richard Feynman (Nobel Laureate in Physics, 1968, for contributions to Quantum Mechanics).

\begin{abstract}
Quantum Education is the "natural" way to learn---motivating and exciting people to take responsibility for their own education.. The Montessori Model represents the closest example of Quantum Education, where the environment is prepared with didactic materials for the children to absorb at their own pace. Where children learn without formal pedagogical machinations, without consciously learning how to learn---by doing. Like Quantum Logic or Quantum Physics or Quantum Games, quantum thinking is an insightful, body/mind approach, attempting to connect our classical world - where objects or things have definite identities-with our new quantum world-where things take on multiple realities simultaneously. The concept of mind, limiting our perceptual abilities is no longer confined to the brain or even the body---all organs are, in some ways, thinking organs, permitting restructuring of our cognitive educational commitment toward infinite choice and possibility. Quantum Education has been defined by the Canadian Quantum 2000 Group as the need for a "quantum" shift in what students are expected to learn in Alberta public schools starting Y2000.
\end{abstract}

\section{Introduction}

A

ttempts to explain Quantum Logic can be both weird and counterintuitive, leading to such unreal statements like "You can have your cake and eat it, too," and "You can be in two places at the same time!" The bizarre state of being in two well-separated places at once can be visualized by imagining a large, shallow roundbottomed bowl with a marble simultaneously at opposite sides of the bowl, rolling from side to side and through itself at the center [2]. The experiment provides a glimpse of quantum superposition states at a scale never seen before, of an ion's single remaining outer electron being in two internal quantum states-"up" and "down"-corresponding to different orientations of the spin of the electron. One Law of Quantum Mechanics allows the electron to be placed in a "superposition" of the two states, in which the two states both exist and are, in a sense, sort of stacked up upon one another. Until the particle is disturbed by an outside agent-a quantum tweak - there is equal probability that it is in either state, and thus it is considered to be in both states.

Quantum-Mechanics theory allows "things" to be in two different places at the same time---weird as that idea may appear to be initially, but think of distant independent on-line learning, or multiple locations for enlightened beings. Quantum thinking changes all our current paradigms---exploding all our artificial limits]. One of the newer goals of education is a wondrous concept called fun---the outward manifestation of that internal state called "fun" is laughter, which Wyndham Lewis called "mind sneezing." Laughter is a unique behavior pattern fully developed only in Homo sapiens.

Physicists argue that the $19^{\text {th }}$ Century was known for the development of machines, the $20^{\text {th }}$ Century for the Information technologies, the $21^{\text {st }}$ Century will be characterized by quantum technologies. This paper attempts to explore quantum relationships in complex humanitas systems; in particular, to characterize, both qualitatively and

Readers with comments or questions are encouraged to contact the authors via email. 
quantitatively, states of entanglement in educational systems, and to champion the quantum approach to education where everybody wins --- learning without conscious learning. Using such mixed quantum/classical concepts as interdependent "entanglement," "super-position" and "frustration," attempt will be made, subsequently, to investigate all phases of the timeline cycle of Quantum Learning---including interconnected learning curves, information retention duration and areas of creative focus. To help define the real altruistic purpose of our educational system: To help people!

Our present learning environment has brought us to the brink of a dangerous, negative worldview. We all construct the future by everything we think, feel and do, but we tend to do it unconsciously, still using a linear concept of Past-Present-Future, with its limited spatial and informational frontiers. The future of Quantum Education is beginning to look more and more like the Montessori philosophy of "education of the senses," or the Lockean model of the mind, which can only be filled by exposure to sensory stimuli and orderly experiences---the recombining and modification of these simple ideas, transformed by cognitive quantum mechanisms into visceral-type learning from the inside out.

To augment persuasion and articulation ability of business school students, stand-up comedy is used (University of Chicago). Song writing, storytelling and improvisation (Vanderbilt University-Owens Management), and for Shakespearean motivation for other management skills at the corporate executive level (Northrup Grumman). Food "chow-down", before and during classes, including pizza and chocolate candy, for relaxation and memory stimulation. The aromatherapy assist to the path of learning, the role of music and subliminal sound---Mozart effect and "silent sound"- and other sensory aids and teaching techniques to activate the audio-visual-kinescopic gateway senses for learning — key for three, but strive for five!

In addition to our five senses--hearing, seeing, tasting, touching, smelling-with which we experience the world directly, labeling the inputs of the world of our five senses [bird, song, music, soft, salty, etc.] is the "sixth sense." Once aware of the sixth sense, we believe there is something within us, some self, which causes this labeling, some "I" which is the seventh sense. In order to become free, in order to understand our true self, [according to Buddha] we need to develop an eighth sense, which allows us to realize the illusory nature of labels and intellectual analysis of the sixth sense, and the concept of "I" of the seventh sense. "When we achieve satori, or enlightenment, we gain the wisdom to hear our true self---the sound of the heart [15]."

What is the mind? How has "mind" been conceived by free-thinkers, philosophers, educators, psychologists, scientists, sociologists, and theologians in the past two-and-a-half thousand years? Hampden-Turner has divided this mind exploration into nine levels, moving from narrower to broader concepts and from earlier to later [6]:

Beginning with historical and religious ideas of mind (including the Greeks, the Yin-Yang and Christianity); [he] then examines different psychoanalytic and existential concepts from Freud and Jung to Kierkegaard and Camus; the physiology of the brain; the creative mind from William Blake to Arthur Koestler; the psycho-social development (H. S. Sullivan, Erik Erikson); language and communication (Bertrand Russell. Korzybski and Chomsky); psycho-biology and cybernetics (Jonas Salk, Bertalanffy, Gregory Bateson); the paradigmatic mind (Marcuse, Kuhn and Catastrophe Theory); and the structure and use of myth (Le'vi-Strauss, the Oresteia and Martin Luther King).

In Chinese the word "mind" is composed of two characters. The top part of the character means sound [literally, the sun rising], the bottom part of the character stands for heart. When we can hear the sun rising in our hearts, we will be free[15]."

We ultimately define ourselves by what we do with crises. The changes we propose for the quantum educational venue---opening ourselves up to one another, building trust-- involve:

13. a redefining of the purpose of education-to help others! 


\section{4. a new cosmic curriculum which inevitably follows this redefinition, and \\ 15. an exploration of emerging paradigms for the interrelationship of other Quantum technologies with students/teachers/ environments.}

Cosmic-type education focuses on connectedness, that we are all related, that there is a relationship between the developing child and the universe, and the child's cosmic timeline experiences in school, linked with the realtime limitless possibilities of cyberspace. The cosmic plan commences with this theme of creation, a sense of one's place in the universe---from the study of nature, which itself is ordered, cyclic, and meaningful. The unfolding sequence of time (spiritual aspects) and the deductive process of construction (scientific mode) remain balanced, as in life. The carbon-dioxide cycle is the most encompassing cycle used by Montessori to demonstrate nature's order [12]. In this cycle, interdependence of life is revealed as plant and animal life connect. Humans, animals and fish breathe in oxygen and breathe out carbon dioxide. Simultaneously, the reverse activity in the carbon cycle is found in plants, keeping the oxygen level almost constant; thus assuring the air's gaseous balance necessary for human survival.

In a recent Physical Review (September 2001) Hayden and Benjamin explain how Multi-Player Quantum Games are played with atoms and photons rather than dice and coins, and how quantum games are contests whose outcomes are governed by the unusual logic of the submicroscopic world, enhanced with cognitive nano-quantum computers. The basic token in a quantum game is a "qubit," a bit of data that is stored in an object such as an atomic nucleus. While a classical coin can only be heads (data value 0 ) or tails (data value 1), a qubit can effectively be both heads (0) and tails (1) at the same time, since the nucleus can be in a combination or super-position of spin-up (0) and spin-down (1). What's more, one can interlink or "entangle" qubits held by separate players so that manipulating one qubit strongly affects the others. More than a diversion, playing quantum games can reveal new information-processing tasks (possibly even certain types of financial transactions) that rudimentary present-day "quantum computers" could perform more efficiently than classical computers. Towards these ends, theorists have been taking traditional games, adapting them for the quantum realm, and checking if new or better strategies emerge for learning. If we define play as fun, and learning as play, we can include most of the electronic and computerized games that so fascinate us today. Because of their connection with new problem-solving skills, quantum games may even come under the umbrella of the Montessori concept of "learning how to learn."

David Perkins, co-Director of Project Zero--a research group at the Harvard School of Education--states, "the world is not made up of well-defined problems. It is made up of complicated, messy, chaotic situations...for which we'd better have the skills to cope." Perkins et al have dubbed this coping ability "intelligence in the wild." Thinking "in the wild" means not just being able to solve a problem, but also recognizing that a problem exists in the first place, what that problem is and how much energy and effort should be invested in solving it. Common sense or "street smarts" is a component of this kind of intelligence, but so are curiosity, open-mindedness and sensitivity to one's surroundings.

"The starting point for a better world," the late Norman Cousins said, "is the belief that it is possible... The wild dream is the first step to reality. It is the direction-finder by which people locate higher goals and discern their higher selves [10]." Starting with this belief that it is possible, Quantum Education sermonizes that only by a deep rooted change in ourselves, followed by changes in our institutions-- social, educational, financial, political, ethical, legal, technological—can we go " where no man has gone before"--- from here to here.

\section{References}

1. Ball, Phillip, "Not a Tangled Web," Nature Science Update, Wednesday, 6 September 2000.

2. Dertouzos, M., What Will Be: How the New World of Information Will Change Our Lives. Harper Edge (Harper Collins), San Francisco, CA 1997.

3. Du, Jiangfeng. et al, "Experimental realization of quantum games on a quantum computer," Physical Review Letters, 88, 137902 (2002).

4. $\quad$ Ferguson, Marilyn, The Aquarian Conspiracy: Personal and Social Transformation in the 1980s. Tarcher: 
Los Angeles, CA, 1980.

5. Gates, William H., Business @ The Speed of Thought: Using a Digital Nervous System. Warner Books, 1999.

6. Hampden-Turner, C., Maps of the Mind: Charts and Concepts of the Mind and Its Labyrinths, Mitchell Beazley: London, 1981.

7. King, Serge Kahili, Urban Shaman: A Handbook for Personal and Planetary Transformation, Simon \& Schuster (Fireside): New York, 1990.

8. Klarreich, Erika, "Playing by Quantum Rules," Nature, vol. 414, No. 6861, 15 November 2001, pp. 244245.

9. McLaughlin, C. \& G. Davidson. Spiritual Politics: Changing the World From the Inside Out, Ballantine Books: New York, 1994.

10. May, Gerald G., Care of Mind, Care of Spirit: A Psychiatrist Explores Spiritual Direction. Harper San Francisco (Harper Collins): CA, 1992.

11. Meyer, David, in Physics News Update, The American Institute of Physics Bulletin of Physics News, No.411, January 19, 1999.

12. Montessori, Maria, Dr. Montessori's Own Handbook, Heinemann, London.

13. Peterson, Ivars, "Quantum Games: taking advantage of quantum effects to attain a winning edge," Science News, v.156, No..21 November 1999, p.334.

14. Phillips, B. S., Worlds of the Future: Exercises in the Sociological Imagination, Merrill Publishing: Columbus, OH, 1972.

15. Shapiro, D. H., Jr. Precision Nirvana, Prentice-Hall, Englewood Cliffs, NJ, 1978.

16. Toffler, Alvin, The Third Wave. William Morrow: New York, 1980.

Notes 